\title{
Comparative flame structure investigation of normal and inverse turbulent non-premixed oxy-fuel flames using experimentally recorded and numerically predicted Rayleigh and OH-PLIF signals
}

\author{
Franziska Hunger ${ }^{1, a, *}$, Meor F. Zulkifli1, ${ }^{1, b}$, Benjamin A. O. Williams ${ }^{\mathrm{b}}$, Frank Beyrau ${ }^{\mathrm{c}}$, \\ Christian Hasse ${ }^{\mathrm{a}}$ \\ ${ }^{a}$ Chair of Numerical Thermo-Fluid Dynamics, TU Bergakademie Freiberg, Germany \\ ${ }^{b}$ Department of Mechanical Engineering, Imperial College London, Great Britain \\ ${ }^{c}$ Lehrstuhl für Technische Thermodynamik, Otto-von-Guericke-Universität Magdeburg, Germany
}

\begin{abstract}
The structure and characteristics of a turbulent inverse and normal oxy-fuel diffusion flame are investigated. Previous investigations reported in the literature looked at flame characteristics of laminar inverse diffusion flames and their differences to normal diffusion flames. Only few investigations are reported for turbulent inverse diffusion flames and they did not compare the results to the corresponding normal configuration.

The present study uses a combined experimental and numerical approach to compare and analyze a turbulent non-premixed inverse oxy-fuel and a corresponding normal flame, both are non-piloted. Measurements were conducted using simultaneously recorded planar Rayleigh scattering and OH-LIF signals. Due to the significant variation of the effective Rayleigh cross section in mixture fraction space and the unknown $\mathrm{OH}$ quenching contributions, a comparison of derived quantities such as temperature and $\mathrm{OH}$ mole fraction is not possible. Therefore, the Rayleigh and OH-LIF signals were incorporated in the LES flamelet/progress variable approach used here. This allows for a direct comparison of experimentally recorded and numerically predicted Rayleigh and OH-PLIF signals for the flame structure analysis, which includes the joint PDF of both quantities. The two flames are compared in terms of the local flame structure. In addition, differences in the mixing field and especially in the location of turbulent/non-
\end{abstract}

\footnotetext{
${ }^{*}$ Corresponding author

TU Bergakademie Freiberg

Chair of Numerical Thermo-Fluid Dynamics

Fuchsmühlenweg 9

09599 Freiberg

Germany

Phone: +49 3731/ 39 4821, Fax: +493731394555

Email address: franziska.hunger@vtc.tu-freiberg.de (Franziska Hunger ${ }^{1,}$ )
} 
turbulent interface are investigated.

Keywords: Rayleigh scattering, OH-PLIF, oxy-fuel, inverse diffusion flame, large eddy simulation 


\section{Introduction}

Oxy-fuel combustion with increased $\mathrm{O}_{2}$ content in the oxidizer ranging from $30 \%$ to $>95 \%$ is increasingly used in industrial applications, e.g. in oxy-coal combustion to aid carbon capture and storage (CCS) or in industrial furnaces for melting metals, minerals or glass. In addition, partial oxidation of hydrocarbons using almost pure oxygen is a new process for producing synthesis gases for chemical and energetic use. In general, oxy-processes potentially offer advantages compared to air combustion, such as increased thermal efficiency, higher processing rates, reduced flue gas volumes and reduced pollutant emissions [1]. Inverse configurations, where the oxidizer enters the reaction chamber through a central nozzle surrounded by the fuel stream is used in oxy-processes e.g. to shield the chamber walls. Inverse flame configurations offer further benefits such as reduced NOx formation, soot production and flame stabilization through partial premixing of the central oxidizer and the surrounding fuel $[2,3]$.

Significant progress has been achieved in understanding turbulent air combustion using experimental and numerical techniques. Compared to this, only a small number of studies on oxy-fuel and/or inverse configurations are published and the understanding is less advanced than for air combustion. Several laminar air inverse diffusion flame (IDF) investigations are reported in the literature. $\mathrm{A} \mathrm{CH}_{4}$-air IDF was first investigated in [4] for stability mapping. The following investigations of IDFs mainly focused on soot or carbon monoxide formation and an overview is given in [5]. In addition, few laminar investigations on oxy-fuel flames are reported, see e.g [6]. Further, the comparison of laminar inverse and normal diffusion flames (NDFs) was studied. Takagi et al. [7] investigated $\mathrm{N}_{2}$-diluted $\mathrm{H}_{2}$ normal and inverse diffusion flames at the same jet and coflow velocity. Mikofski et al. [8] experimentally investigated the flame length in relation to the jet velocity. Zhang et al. [9] identified differences between IDFs and NDFs based on $\mathrm{OH}^{*}$ chemiluminescence in a $\mathrm{CH}_{4}-\mathrm{O}_{2}$ mixture.

Turbulent inverse air flames were investigated focusing on stability, blow-out behavior and re-ignition, e.g. [10]. Only few investigations consider turbulent inverse oxy-fuel flames with a basic diffusion flame structure when issued into an inert coflow or confinement. An investigation in [11] shows the stability mapping of $\mathrm{CH}_{4} / \mathrm{O}_{2}$ normal and inverse diffusion flames, and a recent study by Choi et al. [12] analyzes the stability limits, NOx formation and flame structure of $\mathrm{CH}_{4} / \mathrm{O}_{2}$ flames. 
Whilst some studies of laminar air flames have found that inverse and normal diffusion flames are similar in structure $[4,8]$, other authors have observed differences in the measured temperature in air combustion [7] and in the $\mathrm{OH}^{*}$ chemiluminescence fields in oxy-fuel combustion [9]. To our knowledge, a similar comparison of turbulent normal and inverse diffusion flames has not been published.

In the present investigation, we focus on analyzing the differences between non-piloted IDFs

and NDFs, extending previous laminar studies $[4,7-9]$ towards turbulent oxy-fuel flames. The influence of turbulence on flame structure in both configurations is studied using experimental and numerical data. Simultaneous Rayleigh and OH-PLIF measurements were conducted. The Rayleigh and OH-LIF signals were incorporated in the flamelet/progress variable approach as part of the Large Eddy Simulation (LES) enabling a direct comparison of the signals. This direct comparison is necessary in these particular flames, since the Rayleigh cross section changes throughout the flame and the $\mathrm{OH}$ quenching partners are not known. Hence, deriving physical quantities like temperature from the signals is prone to large errors. These errors can be avoided by computing the signals directly from the simulations, c.f. $[13,14]$ for more details. Further, the Rayleigh ratio evaluated across the field of view and the OH-LIF signal for the flame front serve as good flame structure indicators. The Rayleigh ratio was shown to be sensitive to minor (species) changes in addition to the temperature [14]. The OH-LIF ratio also depends on the temperature and concentration of the main species. Both quantities may thus independently validate the numerical procedure, but joint statistics provide a stricter test for flame structure comparison. The numerical approach is used here to analyze the NDF/IDF pair in detail with regard to mixing field and flame structure.

\section{Experimental Approach}

\subsection{Burner and Flame Details}

The burner used in this study was identical to [14], consisting of two co-flows of $95.5 \mathrm{~mm}$ (oxidizer/fuel co-flow) and $211.56 \mathrm{~mm}$ (shield co-flow) diameter, surrounding a $5 \mathrm{~mm}$ inner diameter nozzle of $0.5 \mathrm{~mm}$ wall thickness and over 100 diameters long to achieve a fully developed flow. The co-flow gases were homogenized and flow-straightened. The nozzle protruded $40 \mathrm{~mm}$ above the co-flow exit plane. To avoid lab air entrainment artefacts, measurements are not 
taken beyond $70 \mathrm{~mm}$ downstream from the nozzle exit. The mixture was chosen to exhibit typical oxy-fuel characteristics like a high $\mathrm{O}_{2}$ content, no $\mathrm{N}_{2}$ dilution and a high adiabatic flame temperature, further having an additional and significant $\mathrm{CO}_{2}$ dilution.Moreover, $\mathrm{O}_{2}{ }^{-}$ rich oxidizer and $\mathrm{H}_{2}$-rich fuel was chosen to avoid soot formation. Finally, a composition of fuel: $17.5 \% \mathrm{CH}_{4}, 40 \% \mathrm{CO}_{2}, 42.5 \% \mathrm{H}_{2}$ and oxidizer: $68 \% \mathrm{O}_{2}, 32 \% \mathrm{CO}_{2}$ (by volume) was used after considering flame stability as well. For more details see [14]. In the NDF, fuel flowed through the nozzle, while oxidizer was supplied to the co-flow. The IDF was generated by inverting this flow arrangement. The shield co-flow contained air and $\mathrm{N}_{2}$ for the NDF and IDF respectively, suppressing a potential secondary IDF flame and with velocity set equal to the inner co-flow gas. In both cases, the velocities of the nozzle and co-flow were maintained at $30 \mathrm{~m} / \mathrm{s}$ and $0.2 \mathrm{~m} / \mathrm{s}$.

\subsection{Simultaneous Rayleigh / OH-PLIF measurements}

The measurement principles used in this experiment have been described elsewhere for Rayleigh [15-17] and OH-PLIF [18, 19]. Simultaneous diagnostics were applied to both flame types. Rayleigh scattering was induced using three spatially overlapped frequency-doubled Nd-YAG cavities (THALES Multi-channel system) producing a combined energy of $1.5 \mathrm{~J} /$ shot at $532 \mathrm{~nm}$. The $Q_{1}(8)$ line of the $A^{2} \Sigma^{+}-X^{2} \Pi(1,0)$ transition at around $283 \mathrm{~nm}$ was excited using a Sirah PrecisionScan dye laser (Rhodamine 6G solution in ethanol) producing around $5 \mathrm{~mJ} /$ pulse in the UV which is well below the saturation regime. The $Q_{1}(8)$ OH-LIF excitation scheme was chosen to minimize ground-state Boltzmann fraction population distribution variation over the expected temperature range; a LIFBASE calculation predicted $<7 \%$ variation [20]. The lasers fired sequentially (30 ns apart for the first three cavities) for Rayleigh measurement, followed by the fourth cavity $430 \mathrm{~ns}$ later to pump the dye laser. Such sequential timing mitigates cross talk between the diagnostics. The joint Rayleigh/OH-LIF measurements ran at $10 \mathrm{~Hz}$ repetition rate and were essentially instantaneous because the elapsed time for a single measurement was orders of magnitude shorter than the flow timescales. Both the UV and visible beams were expanded and focused using a combination of spherical and cylindrical lenses before overlapping using a dichroic mirror to create coincident laser sheets with thickness of approximately $120 \mu \mathrm{m}$ and height of $25 \mathrm{~mm}$ at the center of the imaged region. Stray light was managed using beam stops and matte black screens. 
The Rayleigh scattered light was collected using an unintensified interline-transfer CCD camera (LaVision Imager Intense) fitted with a camera lens $(\mathrm{f} / 1.2, \mathrm{f}=50 \mathrm{~mm})$ and a $532 \mathrm{~nm}$ interference filter $\left(\Delta \lambda_{\mathrm{FWHM}}=3 \mathrm{~nm}\right)$ and set to a $1 \mu$ s exposure duration. Following $2 \times 2$ binning of the $1280 \times 1024$ pixel sensor, an imaging region of $33 \mathrm{~mm}$ x $25 \mathrm{~mm}$ with a resolution of $50 \mu \mathrm{m} /$ pixel resulted. For the OH-LIF, an ICCD camera (LaVision Imager Intense plus IRO) with a $100 \mathrm{~mm}$ Halle AR-coated UV lens (f/2.0) was used to acquire the fluorescence signal peaking around $308 \mathrm{~nm}$. Color glass filters (Schott UG5 and WG305) were used to block elastically scattered light, and the intensifier gate set to $200 \mathrm{~ns}$ to reduce background luminosity. The two camera images were realigned and distortion-corrected in post-processing, with subpixel accuracy. Further post-processing corrected for laser energy fluctuations, camera dark noise, stray light and spatial uniformity using standard approaches.

\section{Numerical Approach}

The numerical approach is only briefly outlined in the following, details can be found in $[14,21]$. The flamelet/progress variable (FPV) approach [22] is combined with the LES. For mass and momentum transport the Favre-filtered equations for the momentum $\bar{\rho} \widetilde{\boldsymbol{u}}$ and the density $\bar{\rho}$ are solved. Sub-grid turbulence is accounted for using the sigma model [23] with a model constant of $C_{\sigma}=1.3$.

\subsection{Flamelet-progress variable approach}

The FPV approach requires an additional transport equation for the conserved scalar mix-

ture fraction $\widetilde{Z}$ and the non-normalized progress variable $\widetilde{Y_{C}}$. In addition, an algebraic equation for $\widetilde{Z^{\prime \prime 2}}$ is solved [22]. The thermo-chemical state is retrieved from flamelet look-up tables, which are built by solving the laminar non-premixed flamelet equations for the temperature and species mass fractions in mixture fraction space [24] applying the GRI-MECH 3.0 mechanism [25]. An in-house flamelet solver is used, which also performs the mapping from the scalar dissipation rate to the normalized progress variable $C=Y_{C} / Y_{C, e q}$, where $Y_{C}=Y_{\mathrm{H} 2 \mathrm{O}}$ was chosen. The filtered density function (FDF)-integration is performed by applying a presumed $\beta$-FDF for the mixture fraction and a $\delta$-FDF for the normalized progress variable, assuming statistical independence [22]. The retrieval from the flamelet look-up table uses a multivariate interpo- 
lation. The interpolation weights are determined using a recursive n-dimensional multi-linear interpolation method optimized for memory placement and computational efficiency [26]. The simulations are performed applying a unity Lewis-number approach, which was shown to be adequate for this mixture for turbulent conditions [14].

\subsection{Computation of LIF and Rayleigh signals}

The predicted Rayleigh ratio and the normalized OH-LIF signal are directly compared to the experimental signals. Both quantities are calculated based on the laminar flamelet solution for species and temperature. To ensure a consistent comparison with the experimental signals, we use a non-density weighted FDF, which can be obtained from the Favre-filtered FDF, for details see [22].

The OH-LIF signals for the $\mathrm{Q}_{1}(8)$ transition of the $A^{2} \Sigma^{+}-X^{2} \Pi(1,0)$ band were calculated using the following expression, which applies to the linear LIF regime with the excited-state decay rate dominated by collisional quenching,

$$
S_{\mathrm{OH}-\mathrm{LIF}} \propto N_{\mathrm{OH}} f_{J} \frac{A}{A+Q},
$$

where $N_{\mathrm{OH}}$ is the $\mathrm{OH}$ number density, $f_{J}$ is the Boltzmann fraction population of the absorbing state with rotational quantum number $J, A$ is the spontaneous emission rate, and $Q$ is the total collisional quenching rate of the excited state. The total quenching rate is the sum of the quenching rates for each of the collisional partners and is a function of the local temperature. Here, the quenching species $\mathrm{CH}_{4}, \mathrm{CO}, \mathrm{CO}_{2}, \mathrm{H}_{2}, \mathrm{H}_{2} \mathrm{O}, \mathrm{O}_{2}, \mathrm{~N}_{2}$ and $\mathrm{OH}$ were considered and the correlation and quenching cross section for $\mathrm{OH}$ according to Tamura et al. [27] were used. The OH-LIF signal is normalized with its local maximum value at a given height above burner to ensure independence from the experimental setup and to have a consistent comparison of experimental and numerical data.

The Rayleigh signal $S_{\mathrm{R}}$ is proportional to the number density and an effective Rayleigh cross-section. The number density is inversely proportional to the temperature. The effective Rayleigh cross-section is determined by species mole fractions and their corresponding Rayleigh cross-sections $\sigma_{\mathrm{R} i}$. For equal comparison, the actual Rayleigh flame signal is normalized with

the Rayleigh signal of pure, cold oxidizer and the reciprocal of this normalized Rayleigh signal, 
henceforth called Rayleigh ratio $R_{R}$, is used

$$
R_{R}=\frac{S_{\mathrm{R}}^{\text {Ox }}}{S_{\mathrm{R}}^{\text {Flame }}}=\frac{T}{T_{\mathrm{Ox}}} \frac{\sum_{i=1}^{n_{\mathrm{Ox}}} X_{i} \sigma_{R i}}{\sum_{j=1}^{n_{\text {Flame }}} X_{j} \sigma_{R j}}
$$

where $R_{R}$ is proportional to the flame temperature, in contrast to the Rayleigh signal. The constant species-specific cross sections for the main species $\mathrm{CO}_{2}, \mathrm{CO}, \mathrm{O}_{2}, \mathrm{CH}_{4}, \mathrm{H}_{2} \mathrm{O}, \mathrm{H}_{2}$ and

significant intermediates $\mathrm{OH}, \mathrm{O}, \mathrm{H}, \mathrm{C}_{2} \mathrm{H}_{4}, \mathrm{C}_{2} \mathrm{H}_{6}, \mathrm{C}_{3} \mathrm{H}_{8}, \mathrm{C}_{2} \mathrm{H}_{2}$ and $\mathrm{CH}_{3}$ are evaluated following [28]. For details, refer to [14, 21], which includes also an error estimation at the species cross sections.

\subsection{Computational domain and boundary conditions}

The turbulent flame is simulated with an in-house LES solver $[14,21,26]$ based on the OpenFOAM ${ }^{\circledR} 2.1$ framework. The jet velocity inflow boundary is based on a simulation of the pipe flow, which provides turbulent time-dependent inflow profiles. The coflow velocity is set to a block profile. The computational domain is a rectilinear grid of $30 \times 30 \times 150 \mathrm{~mm}$ at the jet inlet, enlarging to $80 \times 80 \times 150 \mathrm{~mm}$ at the outlet with a grid resolution of $86 \times 86 \times 800$ cells, globally refined towards the nozzle. The reported statistics are collected after running the simulation for 5 flow through times (FTT) and then time-integrating for a period of 10 FTTs. For radial statistics, azimuthal averaging was performed.

\section{Results and Discussion}

The inverse and normal diffusion flames are operated maintaining both constant jet and coflow velocities. These two flames are a reference pair similar to the laminar flame in [7].

An inverse flame configuration requires the burner to be operated in a globally overstoichiometric mode to obtain a closed flame shape. Further, the flame length is an important characteristic that is mostly influenced by the stoichiometric mixture fraction and the momentum diameter $d^{+}$, combining the ratio of the nozzle fluid to the ambient gas density and the initial diameter, for details see e.g. [29]. The flame length, evaluated with the mean stoichiometric mixture fraction position at the center line, and the flame length predicted with the correlations in $[29,30]$ are higher in the IDF due to a $d^{+}$that is 1.6 times higher than in NDF. 

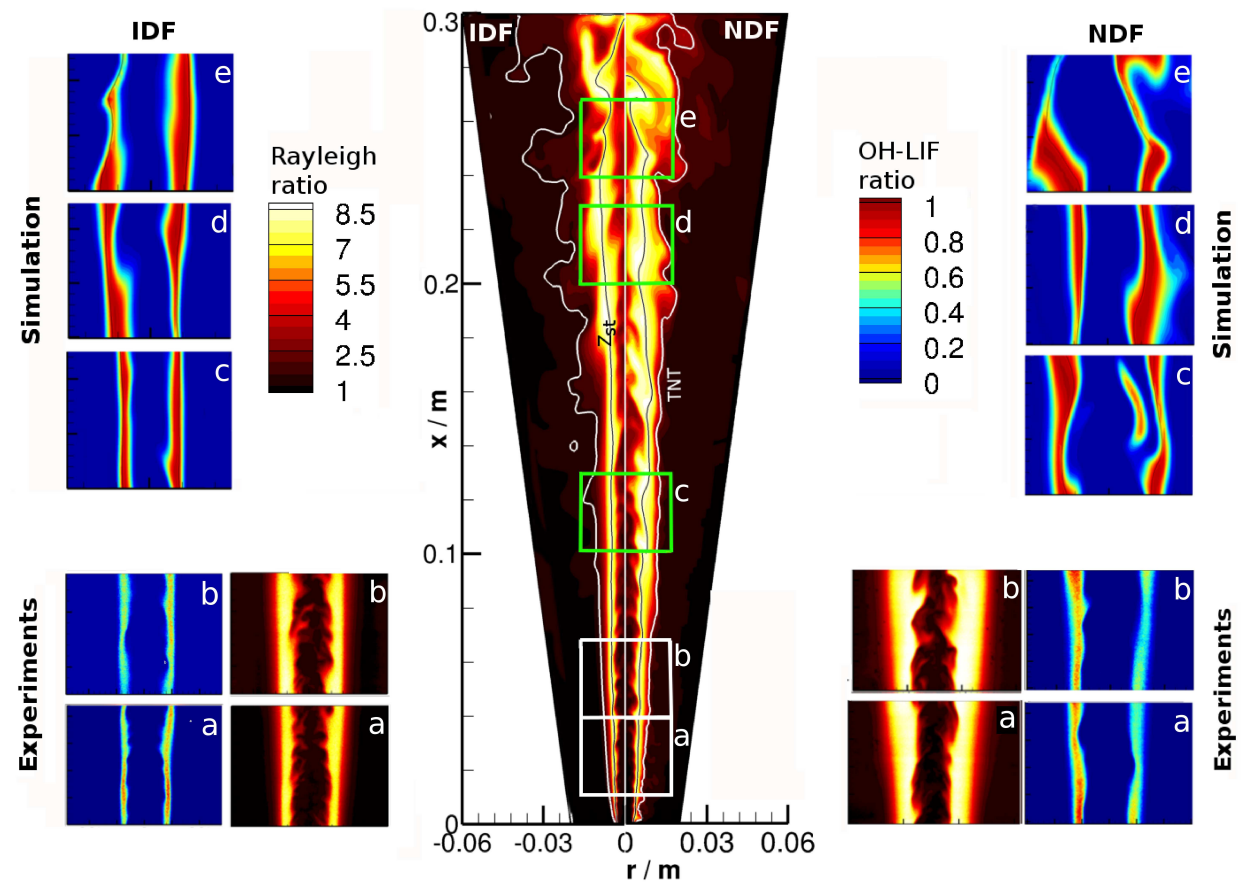

Figure 1: Comparison of instantaneous predicted Rayleigh ratio between IDF (left) and NDF (right) in the computational domain. The lower two white rectangles mark the position where experimental data were recorded and corresponding Rayleigh and OH-LIF data are shown to the left and right $(\mathrm{a}, \mathrm{b})$. The upper three rectangles on each side show the instantaneous predicted OH-LIF ratio in the downstream part of the flame marked in green (c-e). The stoichiometric mixture fraction $\left(Z_{\mathrm{st}}=0.418\right)$ is marked in black and the $\mathrm{T} / \mathrm{NT}$ interface is marked in white $\left(Z_{\mathrm{TNT}, \mathrm{NDF}}=0.05, Z_{\mathrm{TNT}, \mathrm{IDF}}=0.97\right)$. 
Instantaneous Rayleigh and OH-LIF signals for both flames are visualized in Fig. 1. The regions where the experimental data were recorded are marked in white. The experimental setup only allowed measurements to be taken up to $70 \mathrm{~mm}$ downstream of the nozzle. Note that the OH-LIF signal can only be used for a qualitative comparison due to normalization and is thus termed OH-LIF ratio in the remainder. Furthermore, the iso-line of stoichiometric mixture fraction $Z_{\mathrm{st}}=0.418$ and the resolved turbulent/non-turbulent $(\mathrm{T} / \mathrm{NT})$ interface position are marked. Details on the evaluation of the T/NT are given below. Both flames show a broad region of high OH-LIF signal, which is typical for oxy-fuel flames at such high temperatures [6]. Similarly, broad regions of high Rayleigh ratio corresponding to high temperatures are found.

The comparison between the experimentally recorded and numerically predicted Rayleigh and OH-LIF signal of both flames is shown in Fig. 2. Note that only a single height of $67 \mathrm{~mm}$, which is a far downstream location in the experimentally recorded region, is shown as a representative position. These profiles experienced effects of the shear layer generated turbulence, which is captured in the flame simulation and are thus less dependent on the boundary conditions. Similar agreement is found in the other regions upstream. All OH-LIF signals were normalized to the mean OH-LIF peak at this particular height. Good agreement
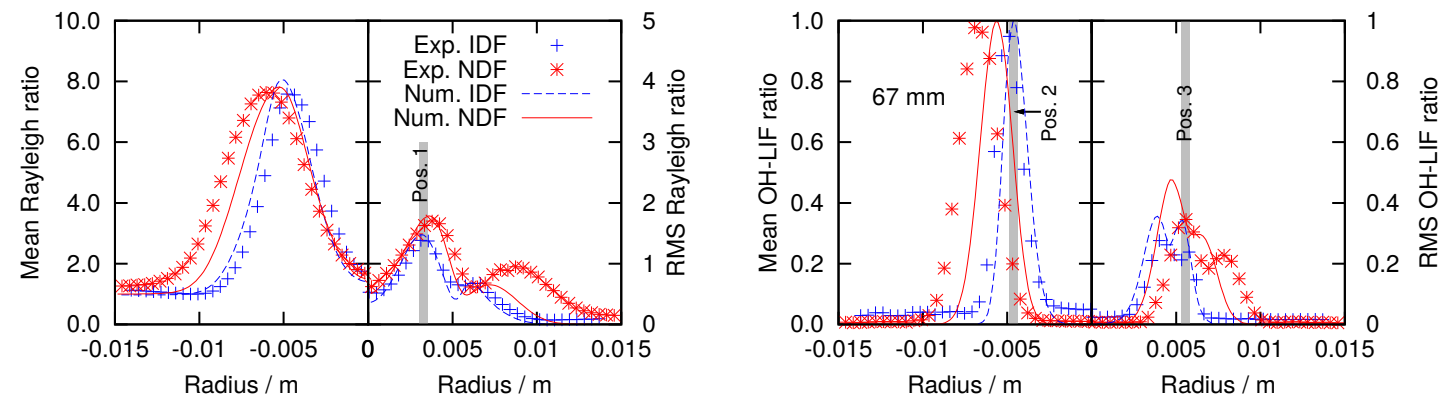

Figure 2: Comparison of the experimental and numerical Rayleigh ratio mean and RMS (left) and OH-LIF ratio mean and RMS (right) at a height of $67 \mathrm{~mm}$ in the NDF and the IDF. The positions where joint PDFs are evaluated are additionally marked.

comparing the experimental and numerical OH-LIF and Rayleigh data is found and this can be stated for the NDF and the IDF. Smaller deviations exist, where the numerics predict a slight shift of the OH-LIF peak towards the burner central axis and the RMS of the OH-LIF signal is overpredicted in the NDF. Next, a comparison of the joint statistics of the two signals 
is performed. Figure 3 shows the joint probability density function (PDF) of the Rayleigh ratio and the OH-LIF ratio at a height of $67 \mathrm{~mm}$ at three different radial positions in the IDF. From left to right these are the position of first Rayleigh RMS peak, the OH-LIF mean peak and the second OH-LIF RMS peak, marked in Fig. 2. These positions are chosen since they exhibit significantly different structures. The first column shows high probabilities in a range

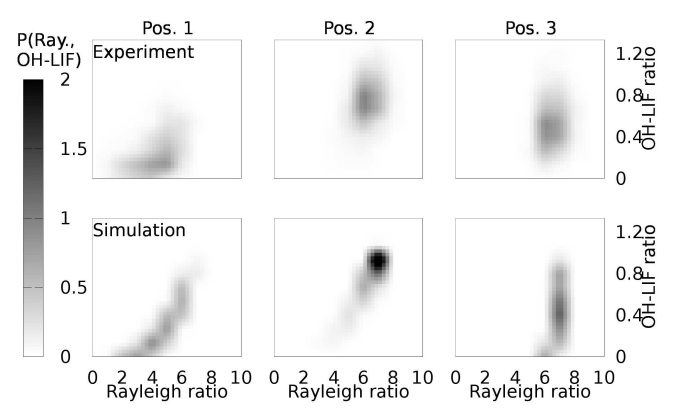

Figure 3: Comparison of the joint PDFs of the experimental data (top row) and the numerical data (bottom row) at the radial positions of the Rayleigh RMS peak, OH-LIF mean peak and OH-LIF RMS peak at a height of $67 \mathrm{~mm}$.

when the Rayleigh ratio and OH-LIF ratio increase almost linearly, which is found for fuel lean mixtures. The second column (OH-LIF peak) exhibits simultaneously high Rayleigh ratios and high OH-LIF signals. In the third column, a high Rayleigh ratio is most probable at a lower $\mathrm{OH}-\mathrm{LIF}$ ratio, which is typical for fuel rich regions. In the numerical data, the existence of higher OH-LIF and Rayleigh ratios (first column) as well as of a lower OH-LIF signal at a high Rayleigh ratio (third column) is also predicted. At the second position, both simulation and numerical data show the same Rayleigh ratio at highest probability. Some differences are visible in the direct vicinity but the overall agreement can be considered good for the joint statistics, which are usually not used for comparing experimental and numerical data. This confirms that the experimental flame structure and its dynamics are captured in the LES. A similar level of agreement was obtained for the joint statistics of the NDF (not shown here).

Furthermore, the comparison of the NDF and the IDF, either based on simulation or experiment, suggests very similar flame structures. However, some differences can be observed, which are investigated next. To quantify the underlying mixing field that directly determines the quantities in physical space, Fig. 4 shows the mixture fraction profiles at a height of $67 \mathrm{~mm}$. The mean mixture fraction $\langle\tilde{Z}\rangle$ for both configurations and $1-\langle\tilde{Z}\rangle$ for the IDF are shown. 


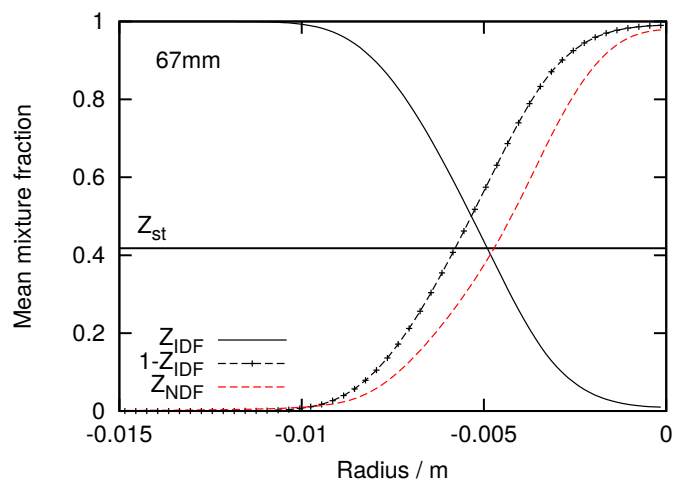

Figure 4: Comparison of mixture fraction profiles at a height of $67 \mathrm{~mm}$. The stoichiometric mixture fraction is additionally marked by a horizontal line.

When comparing $\langle\tilde{Z}\rangle$ from the NDF and $1-\langle\tilde{Z}\rangle$ from the IDF, differences in the jet width are observed, however, stoichiometry is found at almost the same position. This is consistent with the finding that the $\mathrm{OH}-\mathrm{LIF}$ peak (in agreement with the peak of the $\mathrm{OH}$ mole fraction) being in the fuel-lean region, moves towards burner central axis in the IDF.

Next, the flame structure is now investigated in mixture fraction space to understand the underlying flamelet structure. Figure 5 shows the time-averaged species, OH-LIF and Rayleigh ratio profiles at the height of $67 \mathrm{~mm}$ on the right and the time-averaged scalar dissipation rate $\left(\langle\chi\rangle=\left\langle 2\left(D+D_{t}\right)(\nabla \widetilde{Z})^{2}\right\rangle\right)$, normalized progress variable and normalized progress variable source term profiles on the left. The species and signal profiles agree very well in the entire domain. The OH-LIF signals show deviations of less than 5\%. Differences are only visible between $\mathrm{Z}=0.1$ and $\mathrm{Z}=0.5$. The scalar dissipation rate profiles are shifted in mixture fraction space. While the NDF profile's peak is near $\mathrm{Z}=0.5$, the IDF peak is closer to $Z_{\text {st }}$ but with similar maximum values. However, differences are small for the normalized progress variable and this supports the agreement for the resulting species and signal profiles. Notable differences in the progress variable are only visible towards the fuel-lean boundary where the NDF obtains larger values than the IDF. The overall impact of the differences in the progress variable on the flame structure is very small. The almost identical flame structure is an indicator that mixing in both IDF and NDF is very similar in this height when keeping the jet velocity constant.

Further, a very important aspect for understanding the similarity in the underlying flame structure for NDF and IDF is the position of the T/NT. This interface position was determined using the threshold value where the bimodal PDF of mixture fraction in the entire 
computational domain has its minimum according to [31], who investigated the T/NT interface statistics in a non-reacting LES of propane and carbon-dioxide. A threshold value of $Z=0.05$ was found for the NDF and of $Z=0.97$ for the IDF. As visualized in Fig. 1 and marked in Fig. 5 with vertical lines, the T/NT is located far away from stoichiometry, which is contrary to methane-air flames, for example. This is mostly due to the much higher stoichiometric mixture fraction $\left(Z_{s t}=0.418\right)$ here compared to methane air $\left(Z_{s t}=0.055\right)$. Such low values put the stoichiometric surface at the mixing layer edge and thus close to the T/NT interface. This implies that the flamelet structures here are embedded in a fully turbulent flow and no direct influence of the laminar coflow is expected with respect to the flamelet structure near stoichiometry. This is another indication why the influence of differential diffusion is negligible in this flame although the laminar version of the flame is greatly affected by differential diffusion, c.f. [14].

Consequently, the species and measurement signal profiles of IDF and NDF agree very well with each other. Previous investigations on laminar air flames already found structurally similar flames for IDF and NDF $[4,8]$. However, when differential diffusion was strong, differences between IDF and NDF start to emerge, e.g. the temperature profile of the inverse $\mathrm{H}_{2}$-flame [7] departed from the profile of the corresponding normal flame caused by differences in the $\mathrm{H}_{2}$ ratio. Further, the investigation of an inverse and normal $\mathrm{CH}_{4}-\mathrm{O}_{2}$ flame at a constant $\mathrm{O} / \mathrm{C}$ ratio (not comparing flames at the same jet velocity) [9] found significantly different peak $\mathrm{OH}^{*}$ chemiluminescence intensity and profiles in physical space along the burner central axis. For the present turbulent oxy-fuel NDF/IDF pair, the underlying flamelet structure is very similar due to negligible differential diffusion effects. Differences are only caused by different mixture fraction fields.

Since a different mixing field is expected based on the flame appearance in Fig. 1, the mixture fraction as well as the measurement signals are shown further downstream at a height of $250 \mathrm{~mm}$ in Fig. 6. Here, the mixture fraction profiles differ since the centerline values of the NDF more strongly depart from the initial jet value, while stoichiometry is still at the same position. Accordingly, the Rayleigh ratio and the OH-LIF ratio exhibit stronger differences between NDF and IDF than before. Performing the same analysis for the flamelet structure as in Fig. 5 (not shown here), no significant deviations are found indicating that the observed differences are mostly related to the mixing field. 


\section{Conclusions}

A corresponding pair of normal and inverse turbulent oxy-fuel diffusion flames operated at constant jet and coflow velocity was investigated numerically and experimentally.

Experimentally-obtained Rayleigh ratio and normalized OH-LIF data were directly compared to equivalent numerically-predicted signals, by incorporating these observed quantities into the LES/FPV model. Very good agreement was obtained in a comparison of the independent and joint statistics of the inverse and normal diffusion flame.

NDF and IDF were then compared by looking separately at the 3D mixing field and the flame structure conditioned on mixture fraction. The IDF exhibited a longer flame than the NDF. However, especially in the near-field, a qualitative agreement of the mixture fraction field and correspondingly also of the measurement signal profiles was found. Looking at the underlying flamelet structure, the measurement signals as well as the species profiles agree well when conditioned on mixture fraction. Thus, the same general flame structure was identified. One important indication of similar flame structure is that the turbulent/non-turbulent interface for both configurations was found to be far from stoichiometry, meaning that the major part of the flamelet structure is in the fully turbulent regime. Further downstream, notable differences occur, that are mostly related to differences in the mixing field but are not visible in the flamelet structure.

The need to include differential diffusion in flamelet approaches might thus be dependent on the distance between the $\mathrm{T} / \mathrm{NT}$ interface and regions where stoichiometric mixtures are found. The observations made in this flame should be revisited in further investigations.

\section{Acknowledgments}

The authors would like to gratefully acknowledge the financial support by the Saxon Ministry of Science and Fine Arts and the European Union in the framework of the European Social Fund (project number: 100236458).

\section{References}

[1] G. Scheffknecht, L. Al-Makhadmeh, U. Schnell, J. Maier, Int. J. Greenhouse Gas Control 5 (2011) 16 35.

[2] D. M. Stansel, N. M. Laurendeau, D. W. Senser, Combust. Sci. Technology 104 (1995) 207-234. 
[3] C. R. Shaddix, T. C. Williams, L. G. Blevins, R. W. Schefer, Proc. Combust. Institute 30 (2005) 1501 1508.

[4] K.-T. Wu, R. H. Essenhigh, Proc. Combust. Inst. 20 (1985) $1925-1932$

[5] C. R. Shaddix, T. C. Williams, Combust. Flame 156 (2009) $942-945$.

[6] Stelzner, B., Hunger, F., Voss, S., Keller, J., Hasse, C., Trimis, D., Proc. Combust. Inst. 34 (2013) 10451055.

[7] T. Takagi, Z. Xu, M. Komiyama, Combust. Flame 106 (1996) $252-260$.

[8] M. A. Mikofski, T. C. Williams, C. R. Shaddix, L. G. Blevins, Combust. Flame 146 (2006) $63-72$.

[9] T. Zhang, Q. Guo, X. Song, Z. Zhou, G. Yu, J. Spectrosc. 2013 (2013).

[10] A. Elbaz, W. Roberts, Fuel 165 (2016) 447-461.

[11] J. D. Moore, K. K. Kuo, Combust. Sci. Technology 180 (2008) 401-417.

[12] S. Choi, T. Y. Kim, H. K. Kim, J. Koo, J. S. Kim, O. C. Kwon, Energy 93, Part 1 (2015) $1105-1115$

[13] B. Connelly, B. Bennett, M. Smooke, M. Long, Proc. Combust. Inst. 32 (2009) $879-886$.

[14] F. Hunger, M. F. Zulkifli, B. A. O. Williams, F. Beyrau, C. Hasse, Flow Turbul. Combust. 90 (2016) $231-262$.

[15] R. W. Dibble, R. E. Hollenbach, Proc. Combust. Inst. 18 (1981) 1489-1499.

16] A. Eckbreth, Laser Diagnostics for Combustion Temperature and Species, Gordon \& Breach, 1996.

[17] V. Bergmann, W. Meier, D. Wolff, W. Stricker, Applied Physics B 66 (1998) 489-502.

[18] J. Kiefer, Z. S. Li, J. Zetterberg, X. S. Bai, M. Aldén, Combust. Flame 154 (2008) 802-818.

[19] I. Boxx, M. Sthr, C. Carter, W. Meier, Combust. Flame 157 (2010) $1510-1525$.

[20] J. Luque, D. Crosley, LIFBASE: Database and spectral simulation (version 1.5), 1999.

[21] S. Popp, F. Hunger, S. Hartl, D. Messig, B. Coriton, J. H. Frank, F. Fuest, C. Hasse, Combust. Flame 162 (2015) $3016-3029$.

[22] C. Pierce, P. Moin, J. Fluid Mechanics 504 (2004) 73-97.

[23] F. Nicoud, H. B. Toda, O. Cabrit, S. Bose, J. Lee, Phys. Fluids 23 (2011) 085106.

[24] N. Peters, Proc. Combust. Inst. 21 (1986) 1231-1250.

[25] G. P. Smith, D. M. Golden, M. Frenklach, N. W. Moriarty, B. Eiteneer, M. Goldenberg, C. T. Bowman, R. K. Hanson, S. Song, J. William C. Gardiner, V. V. Lissianski, Z. Qin, Gas research institute, http: //www.me.berkeley.edu/gri_mech/version30, 2012.

[26] S. Weise, C. Hasse, Parallel Comput. 49 (2015) 50-65.

[27] M. Tamura, P. A. Berg, J. E. Harrington, J. Luque, J. B. Jeffries, G. P. Smith, D. R. Crosley, Combust. Flame 114 (1998) $502-514$.

[28] F. Fuest, R. S. Barlow, J.-Y. Chen, A. Dreizler, Combust. Flame 159 (2012) 2533-2562.

[29] S. R. Turns, An introduction to combustion. Concepts and Applications, McGrawHil, 2012.

[30] K. M. Tacina, W. J. A. Dahm, J. Fluid Mechanics 415 (2000) 23-44.

[31] M. Gampert, K. Kleinheinz, N. Peters, H. Pitsch, Flow Turbul. Combust. 92 (2014) 429-449. 